\title{
Contralateral disconnection of the rat prelimbic cortex and dorsomedial striatum impairs cue-guided behavioral switching
}

\author{
Phillip M. Baker ${ }^{1,3}$ and Michael E. Ragozzino ${ }^{1,2,3}$ \\ ${ }^{7}$ Program in Neuroscience, University of Illinois at Chicago, Chicago, Illinois 60607, USA; ${ }^{2}$ Department of Psychology, \\ University of Illinois at Chicago, Chicago, Illinois 60607, USA; ${ }^{3}$ Laboratory of Integrative Neuroscience, University of Illinois \\ at Chicago, Chicago, Illinois 60607, USA
}

\begin{abstract}
Switches in reward outcomes or reward-predictive cues are two fundamental ways in which information is used to flexibly shift response patterns. The rat prelimbic cortex and dorsomedial striatum support behavioral flexibility based on a change in outcomes. The present experiments investigated whether these two brain regions are necessary for conditional discrimination performance in which a switch in reward-predictive cues occurs every three to six trials. The GABA agonists baclofen and muscimol infused into the prelimbic cortex significantly impaired performance leading rats to adopt an inappropriate turn strategy. The NMDA receptor antagonist D-AP5 infused into the dorsomedial striatum or prelimbic cortex and dorsomedial striatum contralateral disconnection impaired performance due to a rat failing to switch a response choice for an entire trial block in about two out of 13 test blocks. In an additional study, contralateral disconnection did not affect nonswitch discrimination performance. The results suggest that the prelimbic cortex and dorsomedial striatum are necessary to support cue-guided behavioral switching. The prelimbic cortex may be critical for generating alternative response patterns while the dorsomedial striatum supports the selection of an appropriate response when cue information must be used to flexibly switch response patterns.
\end{abstract}

[Supplemental material is available for this article.]

Changes in environmental contingencies often require a rapid adjustment of actions to achieve goals. Changes in outcome information, such as an empty cache site or cue information, e.g., presence of a predator in a foraging area, represent two fundamental ways in which information is used to guide a switch in actions. In particular, an action that no longer leads to a positive outcome can lead to a subsequent switch in actions. In other conditions, certain cue information may be used proactively to switch actions to obtain a positive reinforcement (Hikosaka and Isoda 2010; Baker and Ragozzino 2014). Numerous studies have demonstrated that various rodent prefrontal cortex and/or striatal subregions support a switch in actions when a particular action is no longer followed by reinforcement in reversal learning or set-shifting tests (Birrell and Brown 2000; Nicolle and Baxter 2003; Tzavos et al. 2004; Kim and Ragozzino 2005; Ragozzino and Rozman 2007; Floresco et al. 2008; McDonald et al. 2008; Kimchi and Laubach 2009; Castane et al. 2010; Pastuzyn et al. 2012). In these reversal learning and set-shifting paradigms, rodents are commonly required to learn an initial discrimination and then either have to reverse choice patterns or learn to use different stimulus information to obtain a reinforcement. Manipulations of different brain areas occur prior to the reversal learning or set-shifting test. The rat prelimbic cortex is one prefrontal cortex area important for set-shifting when there is a change in outcome contingencies, e.g., selecting a choice based on odor information to shifting the choice based on visuospatial information (Birrell and Brown 2000; Ragozzino et al. 2003; Stefani et al. 2003; Rich and Shapiro 2007, 2009; Oualian and Gisquet-Verrier 2010;

\section{Corresponding author: mrago@uic.edu}

Article is online at http://www.learnmem.org/cgi/doi/10.1101/lm.034819.114.
Enomoto et al. 2011; Bissonette and Powell 2012). The set-shifting deficits following prelimbic cortex inactivation result from initial perseveration of the previous response pattern, but do not affect maintaining a currently correct response pattern after an initial switch (Ragozzino et al. 1999a,b; Stefani et al. 2003; Block et al. 2007; Floresco et al. 2008).

Recent studies investigating the prelimbic cortex indicate that this area also supports behavioral switching when cues can be used to shift response patterns for an upcoming choice (Leenaars et al. 2012; Baker and Ragozzino 2014). In these behavioral paradigms, rats commonly learn the different discrimination contingencies prior to manipulations of brain areas. In a cueguided behavioral switch the prelimbic cortex may not only reduce initial perseverative responses, as observed in set-shifting tests, but also support multiple processes to enable a fluid behavioral switch. For example, in learned conditional discrimination tests in which a visual cue signals that a behavioral switch should occur every few trials, e.g., three to six trials, GABA agonists into the prelimbic cortex impaired performance by increasing errors during the initial switch trial, as well as increasing errors immediately following a switch error (perseverative error) and errors after making an initially correct behavioral switch in a trial block (maintenance error) (Leenaars et al. 2012; Baker and Ragozzino 2014). Thus, the prelimbic cortex not only enables behavioral switching when a change in reward outcomes signals a behavioral

(C) 2014 Baker and Ragozzino This article is distributed exclusively by Cold Spring Harbor Laboratory Press for the first 12 months after the full-issue publication date (see http://learnmem.cshlp.org/site/misc/terms.xhtml). After 12 months, it is available under a Creative Commons License (AttributionNonCommercial 4.0 International), as described at http://creativecommons. org/licenses/by-nc/4.0/. 
switch, but also when reward-predictive cues can be used to proactively switch response patterns. Moreover, analysis of the errors committed during trial blocks suggest that the prelimbic cortex may be critical for sustained monitoring of task cues when they signal repeated behavioral switches.

Similar to prelimbic cortex inactivation, NMDA receptor blockade in the subthalamic nucleus impairs cue-guided behavioral switching, as well as a contralateral disconnection of the prelimbic cortex and subthalamic nucleus (Baker and Ragozzino 2014). Contralateral disconnection of the prelimbic cortex and subthalamic nucleus impaired cue-guided behavioral switching by selectively increasing switch and perseverative errors. This differs somewhat from bilateral prelimbic cortex inactivation alone, which increased switch and perseverative errors, but additionally increased maintenance errors. This latter finding raises the possibility that the prelimbic cortex interacts with other brain areas to maintain a behavioral switch after being initially executed. The dorsomedial striatum may be one brain region that, combined with the prelimbic cortex, enables maintenance of a behavioral switch. The dorsomedial striatum receives excitatory input from the prelimbic cortex (Sesack et al. 1989; Conde et al. 1995; Gabbott et al. 2005; Mailly et al. 2013). In tests where a change in reward outcome signals a behavioral switch is required, the dorsomedial striatum supports a switch in response patterns by facilitating the maintenance of the new response pattern (Pisa and Cyr 1990; Ragozzino et al. 2002, Ragozzino 2003; Braun and Hauber 2011). For example, in an egocentric response reversal learning test, NMDA receptor blockade in the dorsomedial striatum impairs reversal learning by selectively increasing maintenance errors (Palencia and Ragozzino 2004). Although there is some evidence that the dorsal striatum also supports cue-guided behavioral switching (Adams et al. 2001; Featherstone and McDonald 2005; Hallock et al. 2013), it is unknown whether the dorsomedial striatum combined with the prelimbic cortex supports cue-guided behavioral switching by enabling maintenance of a behavioral switch. Past studies have primarily focused on studying how individual brain regions contribute to behavioral switching and not how brain areas interact to support behavioral switching. Investigating how different brain areas interact to support cueguided behavioral switching can provide a richer understanding of the neural systems underlying behavioral flexibility.

To determine whether the prelimbic cortex and dorsomedial striatum together are necessary to enable cue-guided behavioral switching, the present experiments used a contralateral disconnection approach to test visual cue-place conditional discrimination performance, as in past studies (Chudasama et al. 2003; Baker and Ragozzino 2014). To investigate the role of the prelimbic cortex and dorsomedial striatum in cue-guided behavioral switching, rats received either the GABA agonists baclofen and muscimol infused into the prelimbic cortex and/or the NMDA receptor antagonist D-AP5 infused into the dorsomedial striatum. Experiment 1 determined whether bilateral baclofen/muscimol infusions into the prelimbic cortex or bilateral D-AP5 infusions impaired conditional discrimination performance. Experiment 2 determined whether contralateral and/ or ipsilateral disconnection of the prelimbic cortex and dorsomedial striatum af- fected conditional discrimination performance. Experiment 3 determined whether pharmacological manipulations of the prelimbic cortex and dorsomedial striatum had a more general effect on discrimination performance that did not require a switch in response patterns. In the visual cue-place discrimination, rats learned to use a visual cue in the start arm of a T-maze to select one of two place locations to receive a food reward. The visual cue (black or white) was switched every three to six trials indicating a rat should enter the other place location. The experiments further determined whether these pharmacological manipulations affected switch trial performance, initial perseveration of a previously relevant response pattern, and/or maintenance of the currently relevant response pattern once selected.

\section{Results}

\section{Histology}

Rats included in the behavioral analysis from the visual cue-place conditional discrimination test were restricted to those who had cannulae placements in the prelimbic cortex and dorsomedial striatum. Figure 1 shows placements of cannula tip locations for the prelimbic cortex (Fig. 1A) and dorsomedial striatum (Fig. 1B) across the three experiments. Prelimbic cortex cannula placements were primarily located $2.7-3.8 \mathrm{~mm}$ anterior to bregma. Dorsomedial striatum cannulae were principally located in the portion of the nucleus located $0.7-1.7 \mathrm{~mm}$ anterior to bregma.

Thirteen rats were excluded from the analyses because of misplacements. In Experiments 1-3, four rats were excluded due to placements outside the prelimbic cortex. All misplacements were anterior to the prelimbic cortex located in the medial orbital subregion. Three of these rats also had misplaced striatal cannulae. Two rats had bilateral cannulae ventral to the dorsomedial striatum in the nucleus accumbens and one had a unilateral cannula in the nucleus accumbens. An additional rat was excluded from analysis due to damage in the prefrontal cortex. There were an additional eight rats excluded from analyses in Experiments 1-3 because of cannula placements outside the dorsomedial striatum. One rat had a unilateral placement in the nucleus accumbens core with another rat having a bilateral
A Prelimbic Area

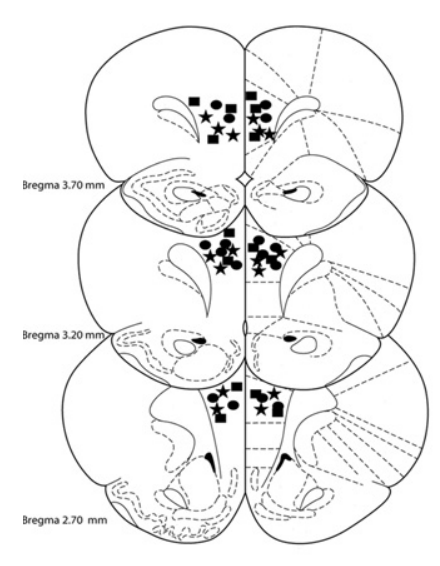

B Dorsomedial Striatum

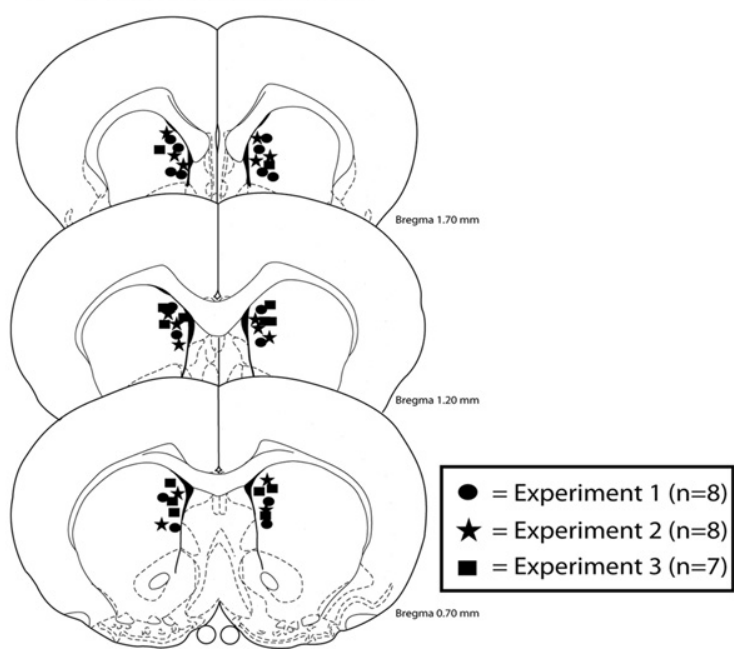

Figure 1. Cannula tip placements in the prelimbic cortex and the dorsomedial striatum in Experiments 1-3. (A) Representation of cannula placements in the prelimbic cortex. (B) Representation of cannula placements targeting the dorsomedial striatum. (Adapted from Paxinos and Watson [1997], with permission from the Anatomical Society of Great Britain and Ireland (c 1997.) 
cannulae placement in the nucleus accumbens core. Two rats had bilateral placements ventral to the dorsomedial striatum in the nucleus accumbens shell. One rat had a bilateral placement in the dorsolateral striatum. Three rats had bilateral placements dorsal to the dorsomedial striatum located in the corpus callosum.

\section{Switch cost in a visual cue-place conditional discrimination}

To determine whether there was a greater likelihood of an error on a switch trial vs. a nonswitch trial, the switch errors and nonswitch errors for vehicle treatment were collapsed across Experiments 1 and 2 . A paired $t$-test revealed that rats were more likely to commit an error on switch trials $(26.00 \% \pm 1.71 \%)$ than on nonswitch trials $(13.34 \% \pm 0.94 \%), t_{(31)}=6.48, P<0.01$.

\section{Experiment 1: bilateral prelimbic cortex inactivation and dorsomedial striatum NMDA receptor blockade impair cue-guided behavioral switching}

Rats $(n=8)$ required $\sim 30$ min to complete a session following the various treatments. The difference in session completion time among the various treatments was not significant, $F_{(3,21)}=0.77$, $P>0.05$.

Vehicle infusions into the prelimbic cortex or dorsomedial striatum led to a performance accuracy of $\sim 80 \%$. In contrast, prelimbic cortex inactivation and NMDA receptor blockade in the dorsomedial striatum reduced conditional discrimination performance to $\sim 65 \%$ (see Fig. $2 \mathrm{~A}$ ). A repeated measures ANOVA revealed that there was a significant treatment effect on performance accuracy, $F_{(3,21)}=49.38, P<0.01$. Post hoc analyses revealed that prelimbic cortex inactivation significantly impaired conditional discrimination performance compared to those of vehicle injections into the prelimbic cortex or dorsomedial striatum $(P$ 's $<0.01)$. In a comparable manner, NMDA receptor blockade in the dorsomedial striatum significantly reduced conditional discrimination performance compared to those of vehicle injections into either the prelimbic cortex or dorsomedial striatum $\left(P^{\prime} \mathrm{s}<\right.$ 0.01 ). There was not a significant difference in performance between prelimbic cortex inactivation and NMDA receptor blockade in the dorsomedial striatum $(P>0.05)$.

The results on the different error measures in Experiment 1 are illustrated in Figure 3. Saline treatment into either brain area led to approximately three switch errors in a session. Prelimbic cortex inactivation increased switch errors to about five per session while D-AP5 infusions into the dorsomedial striatum led to on average four switch errors per session. There was a significant treatment effect for switch errors, $F_{(3,21)}=11.37, P<0.01$. Post hoc analyses revealed that prelimbic cortex inactivation led to significantly more switch errors than the D-AP5 treatment $(P<0.05)$ or either saline treatment $\left(P^{\prime} \mathrm{s}<0.01\right)$. Additionally, dorsomedial striatum NMDA receptor blockade led to an increase in switch errors compared to those of vehicle treatments $\left(P^{\prime} \mathrm{s}<0.05\right)$. Similar to switch errors, there was a significant treatment effect for perseverative errors, $F_{(3,21)}=12.63, P<0.01$. Specifically, NMDA receptor blockade of the dorsomedial striatum led to an increase in perseveration compared to those of all other treatments $\left(P^{\prime} \mathrm{s}<\right.$ $0.01)$. In contrast, no effect of prelimbic cortex inactivation was observed on perseverative errors $(P$ 's $>0.05)$. Finally, there was a significant treatment effect for the number of maintenance errors committed, $F_{(3,21)}=10.93, P<0.01$. Both prelimbic cortex inactivation and dorsomedial striatum NMDA receptor blockade led to an increase in the number of maintenance errors committed compared to those of vehicle treatments $(P<0.01$ and $P<0.05$, respectively), but did not significantly differ from one another $(P>0.05)$.

One limitation of the total error measures is that they do not provide information about the consistency of errors across a test session. For example, a certain error may preferentially occur early in a test session, but not late in a test session or vice versa. An additional analysis on the various error types was conducted to determine whether errors occurred preferentially in the first or second half of a test session. Comparing switch, perseverative, and maintenance errors between session halves revealed that for prelimbic cortex inactivation there was not a significant difference in the number of switch $\left(t_{(7)}=1.32, P>0.05\right)$, perseverative $\left(t_{(7)}=0.00, P>0.05\right)$, or maintenance errors, $t_{(7)}=1.23, P>0.05$. Similarly, for D-AP5 infusions into the dorsomedial striatum there was not a significant difference in the number of switch $\left(t_{(7)}=\right.$ 1.00. $P>0.05)$, perseverative $\left(t_{(7)}=1.07 . P>0.05\right)$, or maintenance errors $\left(t_{(7)}=0.46, P>0.05\right)$ between session halves.

Because the task has several different blocks that vary in length, the total number of errors also does not provide information about the degree to which certain errors occurred. For example, there may be a significant increase in the total number of maintenance errors following a treatment that does not result because such an error was committed across more trial blocks, but because more maintenance errors were committed in a single or small number of trial blocks. To understand the degree to which certain errors occurred, an analysis was carried out to determine the percentage of blocks in which a particular error was committed based on the total number of blocks in which such an error was possible. Analysis of the percentage of error blocks revealed that this measure mimicked the total error pattern for prelimbic cortex inactivation, but deviated to some degree from the total error results for D-AP5 infusions into the dorsomedial striatum. Specifically, there was a treatment effect for percent of switch error blocks, $F_{(3,21)}=11.51$, $P<0.01$. Post hoc tests indicated that prelimbic cortex inactivation significantly increased the percentage of switch error blocks compared to that of saline treatment in the prelimbic cortex or dorsomedial striatum $\left(P^{\prime} \mathrm{s}<0.01\right)$ and 

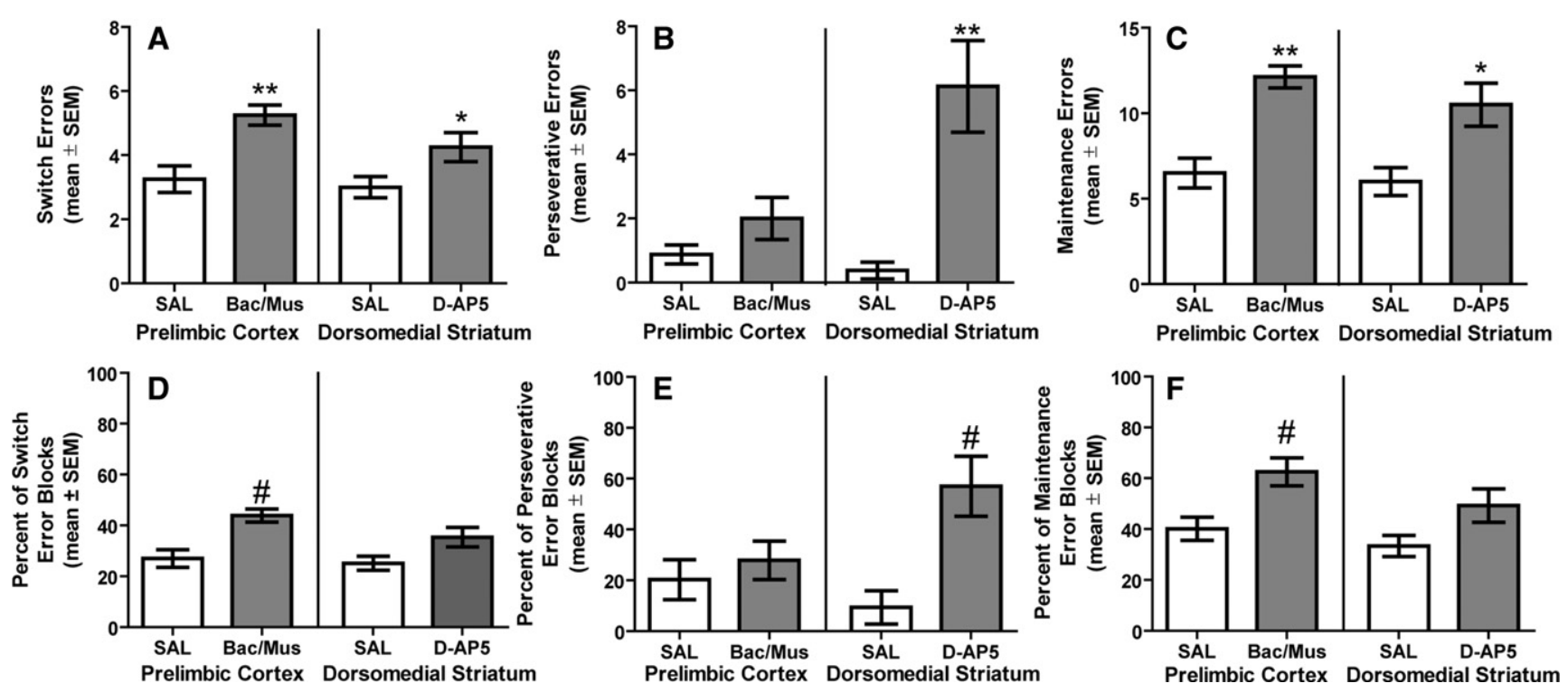

Figure 3. Different errors committed following prelimbic cortex inactivation or dorsomedial striatum NMDA receptor blockade during cue-guided behavioral switching. (A) The total number of switch errors (mean \pm SEM) increased in the Bac/Mus and D-AP5 treatments compared to those of SAL treatments. (**) $P<0.01,\left(^{*}\right) P<0.05$. Additionally, Bac/Mus resulted in a significantly higher number of switch errors than D-AP5 treatment. (B) D-AP5 treatment in the dorsomedial striatum significantly increased perseverative errors compared to those of all other treatments. $\left.{ }^{* *}\right) P<0.01$. $(C)$ The number of maintenance errors increased in the Bac/Mus and D-AP5 treatments compared to those of SAL treatments. $\left({ }^{* *}\right) P<0.01,(*) P<0.05$. (D) Percent of switch error blocks. Bac/Mus treatment in the prelimbic cortex significantly increased the percent of switch error blocks compared to those of SAL treatments and D-AP5 treatment. $\left.{ }^{\#}\right) P<0.05$ vs. D-AP5 and $P<0.01$ vs. SAL treatments. (E) Percent of perseverative error blocks. D-AP5 treatment in the dorsomedial striatum led to a significantly higher percent of perseverative errors than SAL treatments as well as Bac/Mus in the prelimbic cortex. $(\#) P<0.01$ vs. SAL treatments and $P<0.05$ vs. Bac/Mus. $(F)$ Percent of maintenance error blocks. Bac/Mus treatment led to a significantly higher percent of maintenance errors than saline treatment. $\left(^{\#}\right) P<0.05$ vs. prelimbic SAL and $P<0.01$ vs. dorsomedial SAL.

dorsomedial striatum NMDA receptor blockade $(P<0.05)$. However, there was not a significant effect of dorsomedial striatum NMDA receptor blockade on the percentage of switch error blocks compared to that of saline treatment $\left(P^{\prime} \mathrm{s}>0.05\right)$, reflecting a weaker effect that D-AP5 infusions had on switch errors. There was also a significant treatment effect on the percentage of perseverative error blocks, $F_{(3,21)}=5.25, P<0.01$. Post hoc analyses revealed that D-AP5 treatment led to a significantly higher percentage of perseverative error blocks compared with that of saline in the prelimbic cortex $(P<0.05)$ or the dorsomedial striatum $(P<0.01)$. There were no other significant differences between treatments for the percent of perseverative error blocks. Additionally, there was a significant treatment effect on the percentage of maintenance error blocks, $F_{(3,21)}=5.91, P<0.01$. Specifically, baclofen/muscimol injection in the prelimbic cortex resulted in a significantly higher percentage of maintenance error blocks than saline treatment in the prelimbic cortex or dorsomedial striatum $\left(P^{\prime} \mathrm{s}<0.05\right)$ and D-AP5 treatment in the dorsomedial striatum $(P<0.05)$. However, in contrast to results from the total error analysis, there was no significant effect of D-AP5 treatment compared to that of saline for the percent of maintenance error blocks $(P>0.05)$.

Observation of rats under D-AP5 treatment suggested that these rats would occasionally commit errors for an entire block of trials. To determine whether a treatment affected the frequency in which a complete error block occurred, an analysis determined the number of vs. Ipsilateral DRUG. complete error blocks (see Fig. 4A). The results revealed a significant treatment effect on the number of complete error blocks in a session, $F_{(3,21)}=9.45, P<0.01$. Specifically, NMDA receptor blockade in the dorsomedial striatum led to significantly more complete error blocks compared to those of all other treatments $(P$ 's $<0.01)$. There was not a significant difference in the number of complete error blocks between any other treatments $\left(P^{\prime} \mathrm{s}>\right.$ 0.05).

In a recent study (Baker and Ragozzino 2014) we observed that prelimbic cortex inactivation led to a significant turn bias, but not a place bias, in the conditional discrimination test. To determine whether any treatments affected turn or place bias, an analysis was conducted to determine whether a treatment biased a rat to preferentially use an egocentric response strategy (e.g., always turn right) or an allocentric place strategy that was largely
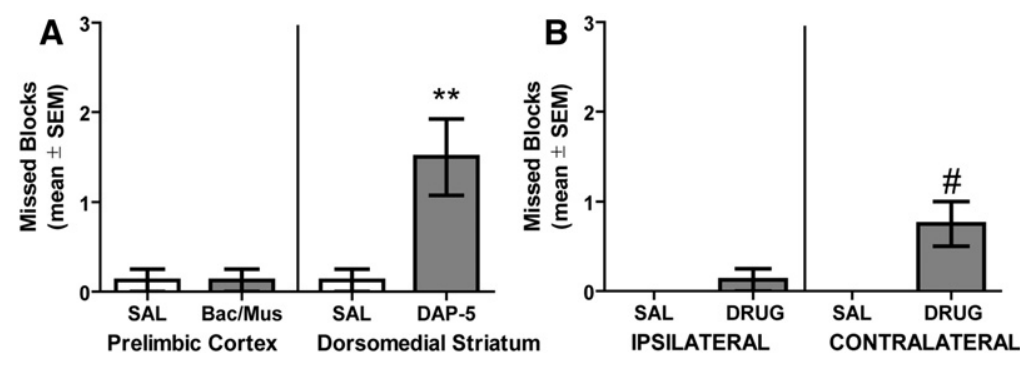

Figure 4. (A) Missed blocks (mean \pm SEM) during the visual cue-place conditional discrimination task. D-AP5 treatment in the dorsomedial striatum led to more missed blocks during a session than all other treatments. $\left.{ }^{(*}\right) P<0.01$ vs. SAL treatments and Bac/Mus. (B) Missed blocks (mean \pm SEM) during the visual cue-place conditional discrimination task. Contralateral treatment led to more missed blocks during a session than all other treatments. (") $P<0.01$ vs. SAL treatments, $P<0.05$ 
independent of the relevant cue-place response (see Supplemental Fig. 1A,B). To determine this, turn bias and place bias scores were measured for each treatment (see Materials and Methods). A turn bias score of 1.00 reflected that a rat always turned in the same direction independent of visual cue or start arm used. A place score of 1.00 reflected that a rat always entered the same maze arm independent of visual cue or start arm used. An examination of turn bias scores revealed that there was a significant treatment effect on turn bias scores, $F_{(3,21)}=5.27, P<0.01$. Prelimbic cortex inactivation $(0.82 \pm 0.03)$ significantly increased turn bias compared to those of prelimbic cortex saline $(0.64 \pm 0.03)$ and dorsomedial striatum saline $(0.63 \pm 0.05)\left(P^{\prime} \mathrm{s}<0.01\right)$ and compared to that of dorsomedial striatal D-AP5 treatment $(P<0.05)$. D-AP5 treatment in the dorsomedial striatum $(0.67 \pm 0.05)$ did not significantly alter turn bias scores compared to those of saline treatments $(P$ 's $>0.05)$. Moreover, there was no significant effect of treatment on place bias scores, $F_{(3,21)}=1.53, P>0.05$.

There were three rats which had prelimbic cortex cannula misplacements in Experiment 1. These rats had a percent accuracy of $80.66 \% \pm 3.84 \%$ following GABA agonist infusion comparable to that of vehicle treatment in rats with accurate placements of $81.37 \% \pm 1.45 \%$. In Experiment 1 , there were eight rats that had misplaced dorsomedial striatum placements. Three of these rats also had prelimbic cortex misplacements. In these eight rats, D-AP5 treatment led to performance accuracy of $85.87 \% \pm$ $1.61 \%$ which was comparable to that of vehicle treatment with accurate dorsomedial striatum placements $(83.62 \% \pm 1.47 \%)$.

\section{Experiment 2: contralateral disconnection of the prelimbic cortex}

\section{and dorsomedial striatum impairs cue-guided behavioral switching}

Following contralateral and ipsilateral treatments, rats $(n=8)$ needed $\sim 30-33 \mathrm{~min}$ to complete a session. A repeated measures ANOVA revealed there was not a significant treatment effect for session time completion, $F_{(3,21)}=2.78, P>0.05$.
The effects of contralateral and ipsilateral disconnections of the prelimbic cortex and dorsomedial striatum areas on conditional discrimination performance are shown in Figure 2B. There was a significant treatment effect for percent accuracy, $F_{(3,21)}=$ $27.99, P<0.01$. Post hoc tests indicated that contralateral disconnection significantly decreased percent accuracy compared to those of contralateral saline treatment $(P<0.01)$ and both ipsilateral saline and drug treatments $\left(P^{\prime} \mathrm{s}<0.01\right)$.

An analysis of errors revealed a significant treatment effect on switch errors, $F_{(3,21)}=4.08, P<0.05$ (see Fig. 5A). The contralateral disconnection treatment significantly increased switch errors compared to those of all other treatments $\left(P^{\prime} \mathrm{s}<0.05\right)$. There was also a significant treatment effect of treatment on perseverative errors, $F_{(3,21)}=17.44, P<0.01$ (see Fig. 5B). The contralateral disconnection treatment significantly increased perseverative errors compared to those of all other treatments $(P$ 's $<0.01)$. Similar to switch and perseverative errors, the difference in maintenance errors among the treatment conditions was significant, $F_{(3,21)}=5.31, P<0.01$ (see Fig. 5 C). The contralateral disconnection treatment led to significantly more maintenance errors than the contralateral saline treatment $(P<0.01)$ and the ipsilateral saline and drug treatments $(P$ 's $<0.05)$.

Examination of errors committed across the test session under contralateral disconnection revealed no significant differences between the first and second half of a session for switch $\left(t_{(7)}=\right.$ $0.31, P>0.05)$, perseverative $\left(t_{(7)}=1.14 P>0.05\right)$, and maintenance errors, $t_{(7)}=0.68, P>0.05$.

As in Experiment 1, an analysis was conducted to determine the degree in which errors occurred in a test session. The results indicated a significant treatment effect for the percent of switch error blocks, $F_{(2,21)}=4.14, P<0.05$. Contralateral disconnection led to a significantly greater percentage of switch error blocks than those of contralateral saline and ipsilateral saline treatments $\left(P^{\prime} \mathrm{s}<0.05\right)$ (see Fig. 5D). There was not a significant difference in percent of switch error blocks between ipsilateral disconnection
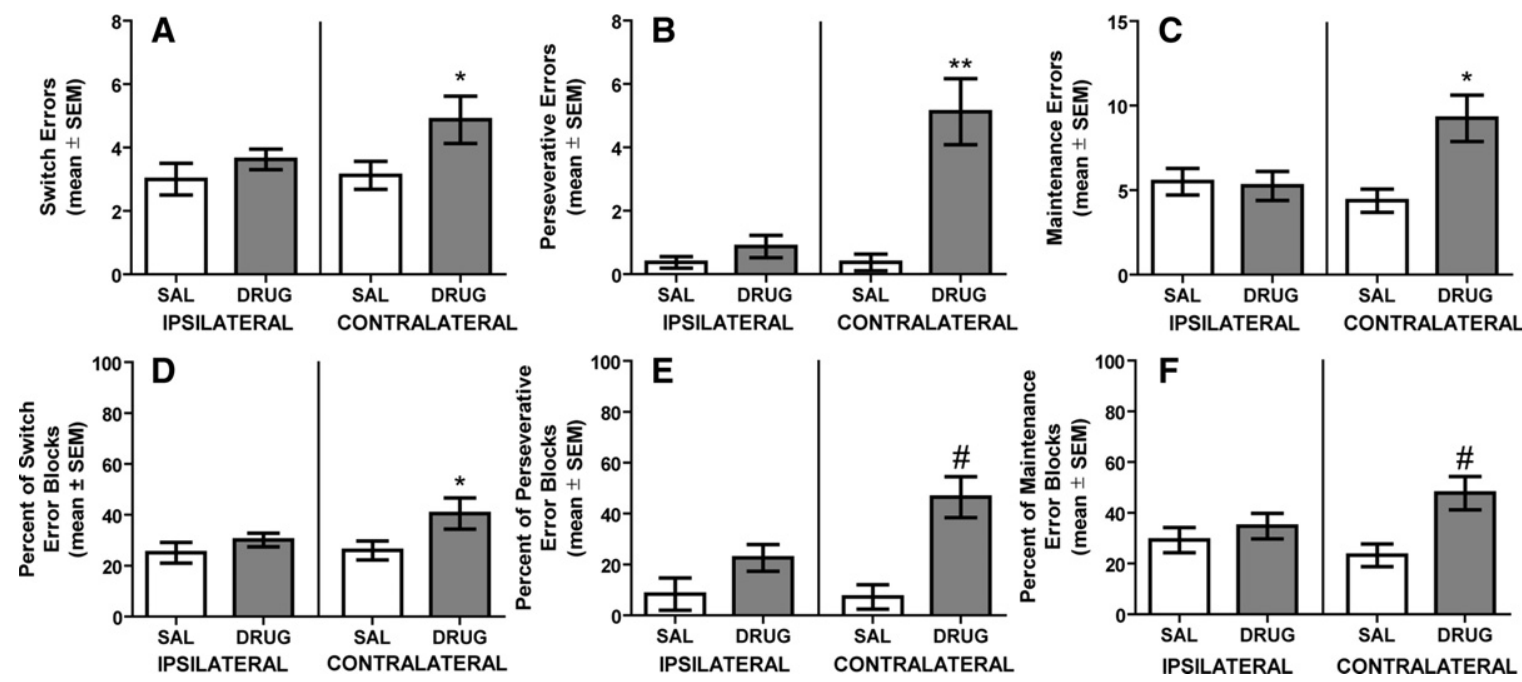

Figure 5. Distribution of errors under disconnection of the prelimbic cortex and dorsomedial striatum during cue-guided behavioral switching. ( $A$ ) The number of switch errors (mean \pm SEM) increased in the contralateral drug treatment compared with other treatments. $\left(^{*}\right) P<0.05$. $(B)$ The contralateral treatment led to significantly more perseverative errors (mean \pm SEM) than all other treatments. $\left.{ }^{* *}\right) P<0.01$. (C) The number of maintenance errors (mean \pm SEM) increased in the contralateral treatment compared to other treatments. $\left(^{*}\right) P<0.05$. (D) Percent of switch error blocks. Contralateral DRUG treatment led to a significantly higher percent of switch error blocks than either SAL treatment. No differences were observed between ipsilateral DRUG treatment and any other treatment. $\left(^{*}\right) P<0.05$ vs. SAL treatments. $(E)$ Percent of perseverative error blocks. Contralateral Drug treatment led to a higher percentage of perseverative error blocks than any other treatment. No differences were observed between ipsilateral DRUG and either SAL treatment. ( $\left.{ }^{\#}\right) P<0.01$ vs. SAL treatments and $P<0.05$ vs. ipsilateral DRUG. $(F)$ Percent of maintenance error blocks. Contralateral Drug treatment led to a higher percentage of maintenance error blocks than contralateral saline treatment. No other differences were observed between treatments. $(\#) P<0.05$ vs. contralateral SAL treatment. 
treatment and any other treatment $\left(P^{\prime} \mathrm{s}>0.05\right)$. There was also a significant effect of treatment on the percent of perseverative error blocks, $F_{(3,21)}=10.95, P<0.01$ (see Fig. 5E). Contralateral disconnection treatment significantly elevated the percentage of perseverative error blocks than either contralateral or ipsilateral saline treatment $\left(P^{\prime} \mathrm{s}<0.01\right)$, as well as ipsilateral disconnection treatment $(P<0.05)$. Moreover, analysis of the percent of maintenance error blocks indicated a significant treatment effect, $F_{(3,21)}=3.82, \quad P<0.05$ (Fig. 5F). Contralateral disconnection treatment significantly increased the percentage of maintenance error blocks compared to that of contralateral saline treatment $(P<0.05)$. There were no other significant differences in the percent of maintenance error blocks between treatments ( $\left.P^{\prime} \mathrm{s}>0.05\right)$.

As illustrated in Figure 4B, there was a significant treatment effect for the number of complete error blocks committed in a session, $F_{(3,21)}=6.86, P<0.01$. Post hoc analyses revealed that contralateral disconnection treatment significantly increased the number of complete error blocks compared to those of saline treatments $\left(P^{\prime} \mathrm{s}<0.01\right)$ or ipsilateral drug treatment $(P<0.05)$.

Contralateral disconnection and ipsilateral disconnection treatments, while affecting the different error measures, did not affect turn or place bias (see Supplemental Fig. 1C,D). The difference in turn bias scores among the treatments was not significant, $F_{(3,21)}=2.47, P>0.05$. In a comparable fashion, there was no significant treatment effect for place bias scores, $F_{(3,31)}=$ 2.07, $P>0.05$.

\section{Initial block performance in the visual cue-place conditional discrimination}

To test whether the conditional discrimination impairments observed with the various treatments were due to a general inability to perform a visual cue-place discrimination and not due to the difficulty of switching repeatedly between blocks of trials, the performance on the initial block of trials was compared among treatments. In Experiment 1, no significant effect of treatment was observed on the 1st block performance, $F_{(3,21)}=2.91, P>0.05$. Likewise, no significant effect of treatment was observed on the 1 st block of trials in Experiment 2, $F_{(3,21)}=0.93, P>0.05$. Thus, the treatments that impaired overall conditional discrimination performance had no effect on initial discrimination accuracy.

Of the three rats that had misplacements in Experiment 2 (all due to misplaced dorsomedial striatal cannulae), performance ranged from $75.33 \% \pm 0.88 \%$ in the contralateral high dose treatment to $85.67 \% \pm 4.41 \%$ with the ipsilateral vehicle treatment, which was also comparable to vehicle treatments with accurate placements.

\section{Experiment 3: prelimbic cortex inactivation, NMDA receptor blockade of the dorsomedial striatum, and contralateral disconnection of the prelimbic cortex-dorsomedial striatum do not impair a nonswitch discrimination test}

To further examine whether treatment effects resulted from a more fundamental deficit in discrimination performance, nonswitch discrimination performance was tested under all effective treatments (Fig. 6). Specifically, the effects of bilateral baclofen/ muscimol infusions into the prelimbic cortex, bilateral D-AP5 infusions into the dorsomedial striatum, and contralateral disconnection of the prelimbic cortex and dorsomedial striatum compared to saline infusions were tested in a nonswitch discrimination performance. In the test, rats $(n=7)$ received a single 28-trial session in which the same visual cue-place contingency was used throughout testing and only switched between sessions. All treatments led to performance with greater than 80\% accuracy. The difference in percent accuracy scores among the treatments

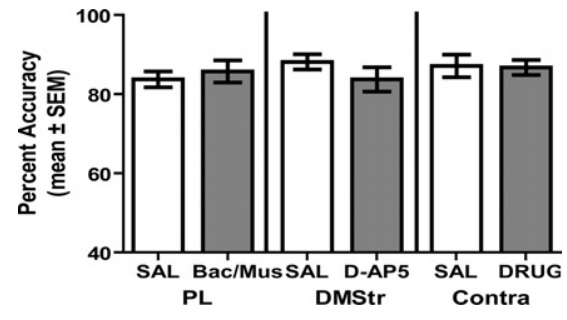

Figure 6. Prelimbic cortex (PL) inactivation, dorsomedial striatum (DMStr) NMDA receptor blockade, and contralateral disconnection of the prelimbic cortex-dorsomedial striatum (Contra) do not affect performance during a nonswitch cued-association test. Each rat $(n=7)$ received six treatments during six separate nonswitch discriminations. Two treatments in the PL were administered; a saline injection (PL SAL), and baclo$\mathrm{fen} /$ muscimol ( $\mathrm{PL}$ Bac/Mus). Two treatments were given in the Dorsomedial striatum; a saline control (DMS SAL), and the NMDA receptor antagonist D-AP5 (Dorsomedial striatum D-AP5). Two treatments were given utilizing a disconnection design to examine the PL-STN areas together; a saline injection (Contra $\mathrm{SAL}$ ), and the previously effective treatment of baclofen/muscimol in the PL and D-AP5 in the Dorsomedial striatum (Contra DRUG). No differences in performance were observed between treatments in a nonswitch discrimination.

was not significant, $F_{(5,30)}=0.52, P>0.05$. Thus, treatments that impaired the conditional discrimination test had no effect in the nonswitch discrimination test.

Two rats were excluded from the analyses because of cannula misplacements in Experiment 3. This was due to misplaced cannulae aimed at the dorsomedial striatum. Performance in these rats was comparable to all other rats in this experiment.

\section{Discussion}

The present studies demonstrated that the prelimbic cortex and dorsomedial striatum are important for behavioral switching when reward-predictive cues proactively signal that a switch in a response pattern should occur. The conditional discrimination test led to establishment of a response set resulting in switch costs in which vehicle treatment doubled the percentage of switch trial errors compared to nonswitch trial errors. This is comparable to that observed in a recent study using the same behavioral procedure (Baker and Ragozzino 2014). Beyond demonstrating that the behavioral test leads to switch costs, the present experiments found that prelimbic cortex inactivation or NMDA receptor blockade impaired cue-guided behavioral switching. Furthermore, contralateral disconnection of the prelimbic cortex and dorsomedial striatum impaired cue-guided behavioral switching. A lack of an effect with the same pharmacological manipulations in rats with misplaced cannula suggests that actions in either the prelimbic cortex or dorsomedial striatum, and not juxtaposed areas, are principally responsible for the behavioral effects. Past evidence suggests that the prelimbic cortex may facilitate memory retrieval when subjects are exposed to salient cues (Botreau et al. 2004). Thus, deficits in cue-guided behavioral switching could have resulted from an impairment in using cue information and/or cue-induced retrieval. However, the same manipulations that impaired cue-guided behavioral switching had no effect on the cued discrimination test that did not require behavioral switching. These findings suggest that the deficits observed with manipulations of the prelimbic cortex and dorsomedial striatum cannot simply be explained by an impairment in memory retrieval. Moreover, because the pharmacological manipulations in either brain region had no effect on the time to complete a session, the conditional discrimination impairment cannot be explained by a more general effect on activity. 
Although pharmacological manipulation of either the prelimbic cortex or dorsomedial striatum alone impaired conditional discrimination performance there was a somewhat distinct error pattern, suggesting that these different brain areas support separate, but complementary, functions to enable cue-guided behavioral switching. In Experiment 1, prelimbic cortex inactivation increased switch and maintenance errors, with a trend toward also increasing perseverative errors. This pattern of errors differs from past findings showing that prelimbic lesions or inactivation selectively increases perseverative errors when a change in outcomes signals a behavioral switch should occur (Dias and Aggleton 2000; Ragozzino et al. 2003; Block et al. 2007). The present study also found that prelimbic cortex inactivation increases in both switch and maintenance errors are not due to a more general impairment in discrimination performance, as prelimbic cortex inactivation did not affect the ability of rats to execute a nonswitch discrimination. The increase in multiple error measures likely reflects prelimbic cortex inactivation biasing rats to use a turn strategy that led to reduced conditional discrimination performance. However, prelimbic cortex inactivation producing a turn bias is task dependent, as the same manipulation did not lead to a turn bias in the nonswitch discrimination test. Previous work indicates that medial prefrontal cortical areas act in a top-down manner during cognitive tasks (Narayanan and Laubach 2006, 2009; van Schouwenburg et al. 2010). One possibility is that the prelimbic cortex, specifically, acts in a top-down manner to coordinate behavioral switching when cues can be used proactively. Neurons in the medial prefrontal areas, including the prelimbic cortex, are known to be modulated by a diverse range of task components during memory and cognitive flexibility tasks, such as previous choices, reward outcomes, and behavioral switches, raising the possibility that similar neural activity is required in these tasks on a trial by trial basis to organize appropriate behavior in response to cues (Bouret and Sara 2004; Horst and Laubach 2012). Coordination of the prefrontal cortex with other areas, such as the striatum and hippocampus during maze-based tasks, is known to be important for organizing behavior (Block et al. 2007; Lee and Lee 2013). For example, naturalistic burst patterns in the prefrontal cortex can drive striatal medium spiny neuron depolarization in vivo. This depolarized state is thought to be important for cueguided behaviors (Gruber et al. 2003; Gruber and O'Donnell 2009). Alternatively, the medial prefrontal cortex phase locks to hippocampal theta rhythms during maze-based tasks important for context specific behaviors (Fujisawa and Buzsaki 2011). Taken together, the findings suggest that under conditions that demand repeatedly switching response patterns, the prelimbic cortex may be critical for monitoring current demands to facilitate a behavioral switch as well as maintaining the current response pattern after the initial switch through connections with multiple regions important for cue-guided switching in a maze task.

Similar to prelimbic cortex inactivation, NMDA receptor blockade in the dorsomedial striatum impaired conditional discrimination performance, but did not affect nonswitch discrimination performance. Besides increasing the number of switch errors, NMDA receptor blockade also significantly elevated the number of perseverative and maintenance errors. This pattern of errors differs from past studies showing NMDA receptor blockade in the dorsomedial striatum selectively increases maintenance errors in behavioral flexibility tests where a change in outcomes signals a behavioral switch (Palencia and Ragozzino 2004, 2006). One possibility is that in the conditional discrimination test, disrupting NMDA receptor signaling in the dorsomedial striatum biases a rat toward the first visual cue-place association exposed in a session. This is because a novel finding from these experiments was that D-AP5 infusions into the dorsomedial striatum increased the probability that a rat would miss an entire block of trials.
This almost never occurred with any of the vehicle treatments or prelimbic cortex inactivation, but occurred almost two times per test session with D-AP5 treatment into the dorsomedial striatum. This same result occurred with contralateral disconnection of the prelimbic cortex and dorsomedial striatum. The likelihood of missing a block of trials was not due to the previous trial block length or the length of the block which was missed. Additionally, the same block type that was missed in its entirety appeared difficult for D-AP5 treated rats in the next exposure of that same block type (e.g., if a black-cued trial block was missed in its entirety the next black-cued trial block led to low performance). Specifically, D-AP5 treatment in Experiments 1 and 2 led to performances of $16 \%$ and $36 \%$ correct in these trial blocks, respectively. These results suggest that a rat was still struggling to override the incorrect behavioral response beyond the block that was missed in its entirety.

One explanation for the increase in missed blocks with dorsomedial striatum inactivation and prelimbic cortex-dorsomedial disconnection is that the change in cue-reward contingencies fails to update such that the originally relevant response pattern continues to be preferentially employed. One possibility is that input from the prefrontal cortex or thalamus initially pauses striatal firing responsible for initiation of the ongoing motor action. This could be accomplished through input onto cholinergic interneurons via NMDA receptors known to facilitate cognitive flexibility (Palencia and Ragozzino 2006; Bradfield et al. 2013). Specifically, cholinergic cell firing has been shown to briefly block cortical input onto medium spiny striatal neurons (Ding et al. 2010). Subsequently, additional input from the prefrontal cortex about cue information is able to generate an alternative action. Without a pause in cortical input onto medium spiny neurons and/or subsequent input from the medial prefrontal cortex about updated choice expectations, the previous motor plan is instead executed, resulting in continual errors even throughout an entire block. Based on the present findings, when NMDA receptor signaling and/or prelimbic cortex input to the dorsomedial striatum is disrupted, this can bias a rat toward selecting the first response pattern executed in a conditional discrimination test session as opposed to appropriately switching to the alternative cue-guided response pattern consistently throughout the test session.

Another important finding was that dorsomedial striatal NMDA receptor blockade increased maintenance errors and also showed a trend to increase the percent of maintenance error blocks, but the latter effect was not significant. The percent of maintenance error blocks effect may be due to the propensity of rats to miss entire blocks of trials under dorsomedial striatum NMDA receptor blockade. Namely, if a rat misses an entire block of trials, after the initial switch trial error all other errors will be counted as perseverative errors. In this case, a rat will not commit a maintenance error in a block. Although NMDA receptor blockade in the dorsomedial striatum did not significantly increase the percent of maintenance error blocks, the overall increase in maintenance errors suggests that this area supports the reliable execution of a recently selected choice pattern under conditions in which cue information can be used to proactively select a response.

Experiment 2 revealed that contralateral disconnection of the prelimbic cortex and dorsomedial striatum also impairs conditional discrimination performance by reducing the ability to shift on switch trials, as well as increasing errors on subsequent trials in a block. The significant increase in perseverative errors following contralateral disconnection resulted, in large part, from an increased likelihood of a rat to miss an entire block of trials. The behavioral deficit following contralateral disconnection of these structures suggests that the prelimbic cortex and dorsomedial striatum are necessary to enable cue-place conditional 
discrimination performance. The connection between the prefrontal cortex and the dorsomedial striatum has been suggested to support action selection (Seo et al. 2012; Wolfensteller and Ruge 2012). Furthermore, a past study using a contralateral disconnection of the prefrontal cortex from the striatum showed that when rats were required to recall their previous choice and then choose the alternative (delayed alternation), contralateral disconnection impaired performance (Dunnett et al. 2005). The current results extend these findings to indicate that the prelimbic cortex and dorsomedial striatum are necessary to correctly switch from an ongoing response pattern to an alternative response pattern based on proactive cue information. Moreover, findings from a rat model of Huntington's disease suggest that plasticity in the prelimbic cortex and dorsomedial striatal circuit is important for behavioral flexibility (Höhn et al. 2011). In homozygous transgenic rats having enhanced CAG repeats, they begin to exhibit behavioral deficits in an auditory-based conditional discrimination test at 4 mo of age that are independent of any motor symptoms. At this same age, stimulation of the prelimbic cortex results in altered paired-pulse facilitation, short-term depression, and long-term potentiation in dorsomedial striatal recordings (Höhn et al. 2011). These results suggest that plasticity in this prefrontal cortex-striatal circuit is critical for the expression of behavioral flexibility as required in cue-guided behavioral switching. Thus, the pharmacologically induced contralateral disconnection of the prelimbic cortex and dorsomedial striatum may have disrupted certain forms of neuronal plasticity in these areas that contributed to the behavioral deficit.

In a recent study, we demonstrated that the prelimbic cortex and subthalamic nucleus are necessary for cue-guided behavioral switching (Baker and Ragozzino 2014). Specifically, NMDA receptor blockade in the subthalamic nucleus was found to impair switching by selectively increasing switch and perseverative errors without affecting maintenance errors. In addition, contralateral disconnection of the prelimbic cortex and subthalamic nucleus also selectively increased switch and perseverative errors. Because the present findings indicated that contralateral disconnection increased maintenance errors, the combined results suggest that the prelimbic cortex interacts with multiple basal ganglia regions to allow a comprehensive and efficient shift for cue-guided behavioral switching. Specifically, excitatory input from the prelimbic cortex to the subthalamic nucleus may generate an inhibition of an ongoing response pattern and selection of an alternative pattern (Baker and Ragozzino 2014). The present data raise the possibility that excitatory input from the prelimbic cortex to the dorsomedial striatum may play a complementary role by enabling the selection and maintenance of an alternative response pattern. Disruption in this neural system could lead to failure in selecting an alternative response and instead lead to continually executing the same, now inappropriate, response pattern. Although the experimental findings suggest that the prelimbic cortex interacts with both the subthalamic nucleus and dorsomedial striatum to facilitate cue-guided behavioral switching, this does not rule out that the prelimbic cortex, or other prefrontal cortex areas, are functionally connected with other basal ganglia areas, i.e., the nucleus accumbens (Floresco et al. 2006a) or intralaminar thalamic nuclei (Canteras et al. 1990; Castle et al. 2005; Brown et al. 2010) to also enhance cue-guided behavioral switching. Future studies can more fully determine the network of brain areas that support cue-guided behavioral switching.

\section{Materials and Methods}

\section{Subjects}

Adult, male Long-Evans rats weighing between 300 and $350 \mathrm{~g}$ at the time of testing served as subjects $(n=35)$. Rats were individu- ally housed in plastic cages $(26.5 \times 50 \times 20 \mathrm{~cm})$ in a temperature $\left(22^{\circ} \mathrm{C}\right)$ and humidity $(30 \%)$ controlled environment and placed on a 12-h light-dark cycle (lights on at 7:00 a.m.). Rats were food restricted to $85 \%-90 \%$ of their ad libitum body weight during the experiment, and water was available ad libitum. Animal care and use was in accordance with the National Institutes for Health Guide for the Care and Use of Laboratory Animals and approved by the University of Illinois at Chicago Institutional Laboratory Animal Care and Use Committee.

\section{Apparatus}

Training and testing occurred in a four arm cross maze made of black acrylic. Maze arms contained a base that was $10 \mathrm{~cm}$ wide $\times$ $55 \mathrm{~cm}$ long, two side walls that were $15 \mathrm{~cm}$ high by $55 \mathrm{~cm}$ long and a back wall that was $8 \mathrm{~cm}$ wide and $15 \mathrm{~cm}$ high. A $10 \times 10-\mathrm{cm}$ square-base piece connected all four arms together. A circular food well $(3.2-\mathrm{cm}$ diameter and $1.6 \mathrm{~cm}$ deep) was located $3 \mathrm{~cm}$ away from the end of each arm. The maze was elevated $72 \mathrm{~cm}$ above the floor in a room with various extra-maze cues.

\section{Surgery}

Prior to behavioral training, all rats underwent stereotaxic surgery for bilateral implantation of guide cannulae aimed at both the prelimbic cortex and dorsomedial striatum. Thus, each rat had a total of four guide cannulae implanted. For surgery, rats received a mixture of ketamine $(100 \mathrm{mg} / \mathrm{kg})$ and xylazine $(10 \mathrm{mg} / \mathrm{kg})$. Twenty-two-gauge stainless-steel guide cannulae (Plastics One) were implanted into the prelimbic cortex at a $15^{\circ}$ angle in the dor$\mathrm{sal} /$ medial plane. The stereotaxic coordinates were $\mathrm{AP}+3.0, \mathrm{ML} \pm$ 1.8, DV $-3.0(\mathrm{~mm})$. For the dorsomedial striatum, cannulae were implanted at a $15^{\circ}$ angle in the anterior/posterior plane. The stereotaxic coordinates were $\mathrm{AP} 0.0, \mathrm{ML} \pm 2.0, \mathrm{DV}-3.9$. Cannulae were implanted at an angle to allow for all four cannulae to reach their target areas and allow room for dummy cannulae/dust caps when not being injected. The coordinates for each area were based on the stereotaxic atlas by Paxinos and Watson (1997). Four jeweler screws were positioned in the skull surrounding the cannulae and secured with dental acrylic (Stoetling). During the surgical procedure, meloxicam $(1 \mathrm{mg} / \mathrm{kg})$ was administered to manage pain post-operatively. Rats recovered for $7 \mathrm{~d}$ after surgery before commencing behavioral training. For $5 \mathrm{~d}$ following surgery, rats were fed ad libitum and subsequently food restricted as described above. Following this period, subjects were handled $\sim 10$ min per day.

\section{Training}

Behavioral training in the cue-place conditional discrimination task began a week after surgery in multiple phases. In the first phase, a rat was allowed to consume a quarter piece of Froot Loops cereal (Kelloggs) in each food well. A rat was also picked up after consuming cereal pieces to acclimate being handled in the maze as in past studies.

The second training phase required rats to learn that a visual cue in the stem arm indicated which one of two choice arms to enter for a cereal reinforcement. A black plastic block was placed in one maze arm giving the maze a T-shape. The stem arm served as the start arm and the other two arms served as choice arms. The choice arms remained the same throughout training and testing. The other two arms served as start arms and were switched pseudo-randomly such that the same arm was used a maximum of two consecutive trials. Acrylic inserts that covered the walls and floor of the stem arm served as the visual cues. Black and white inserts were used as the visual cues. Each visual cue was always associated with one maze arm containing a cereal reinforcement. For example, if the cue was white, the reinforcement would be in the north arm, while a black cue indicated the reinforcement would be in the south arm. The location of reinforcement for each cue was counterbalanced across rats. When a rat entered one of the choice arms, it was allowed to travel down the arm and explore the food well. If the choice was correct, it was allowed to consume 
a cereal piece after which it was picked up and placed on top of its home cage. The home cage was placed on a table adjacent to the maze. If an incorrect choice was made, a rat was allowed to proceed to the food well and examine it after which it was picked up and returned to its home cage. A rat was exposed to a single cue in a 28-trial session in this training phase. One session was given every day so that a rat saw the same visual cue every other day (session). Visual cues were alternated each session until a rat achieved at least $80 \%$ reinforcement on two consecutive days.

In the third phase of training, rats received both cues on a single daily session. Rats were trained for 10 consecutive trials with each cue presented in alternating blocks for a total of 40 trials. Across sessions, the cue that was presented first in a session was randomized. After a rat achieved at least $80 \%$ correct for both black and white cue trials in a session, each visual cue block was reduced to five consecutive trials over a total of 40 trials (or eight blocks of alternating cues). A rat had to achieve a minimum of $80 \%$ correct for each cue type to advance to the final training phase. Rats required seven to 16 sessions to reach criterion in this phase.

In the fourth and final training phase, a rat was tested for 57 trials in which a cue was switched every three to six trials. This involved a total of 12 switches in a session and each rat received three blocks each of three, four, five, or six consecutive trials with an extra three trials at the end for the 12th switch. A 57-trial session contained approximately an equal number of presentations for each visual cue (28 or 29). A rat achieved criterion when it accurately discriminated $80 \%$ or greater for each visual cue trial type across a 57-trial session. This phase required one to three sessions for rats to reach criterion. After achieving criterion, the test phase began.

In the conditional cue-place association, the visual cue was changed every three to six trials indicating that a behavioral switch should occur for the upcoming response. The relatively short block length was chosen in order to emphasize the need to monitor task cues on every trial while also having a rat establish a response pattern prior to a switch. This is common in a proactive switch task in order to incur a switch cost such that performance is more difficult on a switch trial compared to those of nonswitch trials (Konishi et al. 2005; Hyafil et al. 2009; Hikosaka and Isoda 2010).

\section{Microinfusion procedure}

Five minutes prior to a test session, a rat received an intracranial infusion. Infusions were delivered via 28-gauge injection cannulae which extended $1 \mathrm{~mm}$ below the guide cannulae. The injection cannulae were connected by polyethylene tubing to a $10-\mu \mathrm{L}$ syringe (Hamilton Company). An infusion into the prelimbic cortex consisted of either saline or GABA agonists baclofen and muscimol (Sigma Aldrich). An infusion into the dorsomedial striatum consisted of either saline or the NMDA antagonist D-AP5 (Tocris). An infusion into the prelimbic cortex or subthalamic nucleus alone occurred bilaterally with a total volume of $0.25 \mu \mathrm{L}$ at a rate of $0.15 \mu \mathrm{L} / \mathrm{min}$ by a microinfusion pump (74900 Series Cole Palmer). Injection cannulae were left in place for an additional minute following the injection to allow for diffusion. A similar procedure was used for the contralateral and ipsilateral injection procedures except that a unilateral infusion was made in each brain region. Prior to testing, rats remained in their home cages for 5 min after completion of the injection procedure to allow for the drug to take effect (Hikosaka and Wurtz 1985; Krupa et al. 1999; Palencia and Ragozzino 2004; Baker and Ragozzino 2014). Past studies have shown that microinjections of GABA agonists or glutamate antagonists into specific brain structures can act within a couple of minutes to decrease neural activity and last well over 40 min (Kawabe et al. 2008; McMullan and Pilowsky 2012). As in past studies (McCool et al. 2008; Brown et al. 2010), the day prior to the first test procedure, an injection cannula was lowered into each guide cannula and left in place for $2 \mathrm{~min}$. This ensured that any effects observed on the first test day of testing were not due to the initial acute damage caused by the injection cannulae extending $1 \mathrm{~mm}$ beyond the guide cannulae.

\section{Switch costs in a visual cue-place conditional discrimination}

The conditional discrimination test required rats to establish a response based on learned visual cue-place associations and use cue information proactively to switch a response choice. If the procedure led rats to establish a response pattern within a block, then this should lead to a greater switch cost as displayed by a larger percentage of switch errors compared to nonswitch errors. To determine this, performance in the visual cue-place discrimination test was examined in the vehicle treatment for the percentage of switch trial errors committed vs. the percentage of nonswitch trial errors across experiments. In a test session, the switch error percentage was based on a total of 12 switch trials and the nonswitch error percentage was based on the remaining 45 trials.

\section{Experiment 1: the effect of bilateral prelimbic cortex inactivation and dorsomedial striatum NMDA receptor blockade on performance of a visual cue-place conditional discrimination}

Upon completion of training, rats in Experiment 1 were given one of four treatments to examine the role of the prelimbic cortex and NMDA receptors within the dorsomedial striatum in the visual cue-place conditional discrimination. Five minutes prior to a test session, a rat received a bilateral infusion of either saline (SAL), baclofen $0.05 \mu \mathrm{M}$-muscimol $0.18 \mu \mathrm{M}$ (Bac/Mus) into the prelimbic cortex, or saline (SAL) or D-AP5 $10 \mu \mathrm{M}$ (D-AP5) into the dorsomedial striatum. The drug dose for the prelimbic cortex was chosen because we recently showed it sufficient to impair performance on the cue-place conditional discrimination task. The dose of D-AP5 infused in the dorsomedial striatum was used because a similar dose infused into the subthalamic nucleus impaired performance in the same conditional discrimination test (Palencia and Ragozzino 2004, 2006). The order of treatments administered was counterbalanced across rats. Each rat received each treatment with a minimum of $2 \mathrm{~d}$ between test sessions. The day after testing, a rat received no testing. The following day, each rat received a test session, but did not receive an intracranial infusion prior to the test. This procedure was carried out to ensure that there were no lasting effects of a given treatment on the rat's ability to discriminate between the cues. If a rat was unable to perform the discrimination with at least $80 \%$ accuracy on each cue, additional sessions were given until criterion was achieved (no rats required additional sessions in this study). Once a rat had demonstrated the ability to discriminate accurately, the following day another test was performed. This procedure continued until a rat received all four treatments. There were a total of eight rats included in the analysis for this experiment.

For each test session, the percent correct was determined along with an analysis of the errors committed. Similar to past behavioral switching studies (Dias and Aggleton 2000; Floresco et al. 2006b; Brown et al. 2010; Baker et al. 2011; Mohler et al. 2012; Baker and Ragozzino 2014), the error analysis for each block of trials was calculated to determine whether a treatment affected the initial switch, perseveration of the previously correct response after the switch, and/or the inability to maintain the currently correct response. A switch error was defined as a rat failing to initially switch to the currently relevant response when the visual cue changed. Perseverative errors were only committed in a block in which an initial switch error occurred. Specifically, perseverative errors were committed when any subsequent errors were made after a switch error and prior to making a correct response in that block. Thus, the number of possible perseverative errors varied session to session and from rat to rat. Once a rat successfully switched from the previous response to the currently relevant one, it was no longer possible to commit a perseverative error. However, if a rat made a correct response in a block and reverted back to the other response choice in that same block, then this constituted a maintenance error. Again, because when a rat committed a switch or perseverative error varied, the total possible number of maintenance errors varied. Each error type was then summed across the different trial blocks to calculate the total errors for switch, perseveration, and maintenance. 
In addition to determining the different error types, a corrected score based on the percent of blocks in which a given error type occurred was also measured. Because the task has several different blocks that vary in length, the total number of errors does not provide information about the degree to which certain errors occurred. For example, there may be a significant increase in the total number of perseverative errors following a treatment that does not result because such an error was committed across more trial blocks, but because more perseverative errors were committed in a single or small number of trial blocks. To understand the degree to which certain errors occurred, an analysis was carried out to determine the percentage of blocks in which a particular error was committed based on the total possible blocks in which such an error was possible. Specifically, the percent score was based on the total number of blocks in which a particular error occurred divided by the total number of possible blocks in which a particular error could occur. For perseverative errors, a perseverative error could only occur in blocks in which a switch error was committed. For maintenance errors, an error could only occur when a correct choice was made prior to the last trial in a block. For example, if a rat only committed three switch errors and made a perseverative error in two of these blocks then the percent of perseverative error blocks would be $66.7 \%$.

Another possibility is that errors occurred differentially across the test session. This could be due to interference building up as the session continues or due to the rat requiring several blocks to regain the task contingencies. To address these possibilities, each error type under effective treatments was divided into the first and second half of a given session for comparison. Another possibility is that the effective treatments slowed performance of the task, contributing to any differences observed. Overall time to complete a session was compared between treatments to address this.

To determine whether pharmacological manipulations of the prelimbic cortex and/or dorsomedial striatum produce a conditional discrimination deficit that biases a rat to preferentially use an egocentric response strategy (e.g., always turn right) or an allocentric place strategy that was largely independent of switch, perseveration, or maintenance errors, turn bias and place bias scores were calculated for each treatment. Turn bias scores were calculated by determining a percentage of the number of errors committed to the more common egocentric response divided by the total number of errors. For example, if a rat made a total of 10 errors and nine resulted because a rat turned left when it should have turned right into the correct location, then it would have a percent bias score of $90 \%$. Likewise, a place bias score was calculated by determining a percentage of errors for the more common place location divided by the total number of errors. For example, if a rat made a total of 10 errors and eight resulted because a rat entered the south arm when it should have entered the north arm, then it would have a percent bias score of $80 \%$. Finally, a measure of the number of times that a rat missed an entire block of trials during a test session was also recorded to determine the frequency a rat failed to switch for an entire trial block.

\section{Experiment 2: the effect of contralateral disconnection and ipsilateral disconnection of the prelimbic cortex and dorsomedial striatum on performance of a visual cue-place conditional discrimination}

To determine whether a bilaterally intact prelimbic cortex and dorsomedial striatum are necessary for cue-guided behavioral switching, a contralateral disconnection of the two brain areas was carried out. An ipsilateral disconnection of the prelimbic cortex and dorsomedial striatum served as a control. The test procedure was the same as described in Experiment 1. A separate group of rats was tested in this experiment with a total of eight rats included in the final analysis. Each rat received four injections in total. The injections were counterbalanced for hemisphere injected as well as treatment received across rats. A maximum of two injections through any one cannula was administered for each rat. The contralateral disconnection manipulation involved a unilateral infusion into the prelimbic cortex and a unilateral infusion into the opposite hemisphere of the dorsomedial striatum. Doses for each brain area remained the same as in Experiment 1. Contralateral disconnection treatments were: (1) contralateral vehicle injection of saline (CONTRALATERAL SAL); (2) prelimbic baclofen/muscimol and dorsomedial striatum drug doses (CONTRALATERAL DRUG). The ipsilateral disconnection manipulation involved a unilateral infusion into the prelimbic cortex and a unilateral infusion into the same hemisphere of the dorsomedial striatum. Treatments were as follows: (1) prelimbic-dorsomedial striatum injection of saline (IPSILATERAL SAL); (2) ipsilateral injection of the prelimbic cortex and dorsomedial striatum drug doses (IPSILATERAL DRUG). The order of injections and days between test sessions was the same as described in Experiment 1. For all rats, there was a 2-d interval between test sessions. All outcome measurements were the same as in Experiment 1.

\section{Experiment 3: the effect of prelimbic cortex inactivation, NMDA receptor blockade of the dorsomedial striatum, or contralateral disconnection of the prelimbic cortex-dorsomedial striatum in a nonswitch cued-association test} If pharmacological manipulation of the prelimbic cortex, dorsomedial striatum, or contralateral disconnection of these structures impairs conditional discrimination performance, this may result because of a basic impairment in discrimination performance. To determine this, another group of rats was tested in a discrimination task in which only one of the cues was presented throughout a given session. The training procedure was similar to that described above except that training was limited to the procedure in which rats receive a single visual cue per session. Thus, rats were trained to discriminate between the different visual cues but this occurred across sessions and not within a session. Once rats completed two consecutive days of training at $80 \%$ or higher accuracy, they were advanced to the test phase. The test was identical to the training phase in that rats were tested on a single visual cue discrimination for 28 trials. Rats received a total of six intracranial injections in this experiment with a total of seven rats included in the final analysis. Each visual cue was used for three test sessions. The order of treatments was counterbalanced across rats. For all rats, there was a 2-d interval between test sessions. Each rat received the following treatments: (1) bilateral saline infusion into the prelimbic cortex (PL SAL); (2) bilateral baclofen/muscimol high dose infusion into the PL (PL DRUG); (3) bilateral saline infusion into the dorsomedial striatum (DMStr SAL); (4) bilateral D-AP5 high dose infusion into the dorsomedial striatum (DMStr DRUG); (5) contralateral saline infusion into the prelimbic cortex and dorsomedial striatum (CONTRA SAL); and (6) contralateral baclofen/muscimol high dose infusion into the PL and D-AP5 high dose infusion into the dorsomedial striatum (CONTRA DRUG). The same procedure was employed for the interval between test sessions as described in Experiment 1.

\section{Histology}

After completion of behavioral testing, rats were given an overdose of sodium pentobarbital. Rats were intracardially perfused with $0.9 \%$ phosphate buffered saline followed by $4 \%$ formaldehyde solution. The brain was removed and stored in formaldehyde until sectioning. Brains were frozen and cut into $50-\mu \mathrm{m}$ coronal sections on a cryostat. Sections were immediately mounted on slides, dried, and then stained with cresyl violet. Placements were then verified with reference to the stereotaxic atlas of Paxinos and Watson (1997).

\section{Statistical analysis}

In Experiments 1-3, a repeated measures ANOVA for treatment was used to test the effects of drug treatments on performance accuracy, switch errors, perseverative errors, and maintenance errors. Turn bias scores, place bias, and missed block frequency were also analyzed with repeated measures ANOVAs. A significant treatment effect was followed by Tukey's HSD post hoc tests to determine significant differences between treatments. Switch cost and errors divided into halves of a test session were analyzed 
by using paired Student's t-test comparing percent error rates on switch vs. nonswitch trials and the various error types, respectively.

\section{Acknowledgments}

This research was supported by National Institutes of Health Grant P50 HD055751 to M.E.R. P.M.B. was supported by the University of Illinois at Chicago Center for Clinical and Translational Science (CCTS), Award Number UL1TR000050, from the National Center for Advancing Translational Sciences, National Institutes of Health.

\section{References}

Adams S, Kesner RP, Ragozzino ME. 2001. Role of the medial and lateral caudate-putamen in mediating an auditory conditional response association. Neurobiol Learn Mem 76: 106-116.

Baker PM, Ragozzino ME. 2014. The prelimbic cortex and subthalamic nucleus contribute to cue-guided behavioral switching. Neurobiol Learn Mem 107: $65-78$.

Baker PM, Thompson JL, Sweeney JA, Ragozzino ME. 2011. Differential effects of 5- $\mathrm{HT}_{2 \mathrm{~A}}$ and $5-\mathrm{HT}_{2 \mathrm{C}}$ receptor blockade on strategy-switching. Behav Brain Res 219: 123-131.

Birrell JM, Brown VJ. 2000. Medial frontal cortex mediates perceptual attentional set shifting in the rat. J Neurosci 20: 4320-4324.

Bissonette GB, Powell EM. 2012. Reversal learning and attentional set-shifting in mice. Neuropharmacology 62: 1168-1174.

Block AE, Dhanji H, Thompson-Tardif SF, Floresco SB. 2007. Thalamicprefrontal cortical-ventral striatal circuitry mediates dissociable components of strategy set shifting. Cereb Cortex 17: 1625-1636.

Botreau F, El Massioui N, Cheruel F, Gisquet-Verrier P. 2004. Effects of medial prefrontal cortex and dorsal striatum lesions on retrieval processes in rats. Neuroscience 129: 539-553.

Bouret S, Sara SJ. 2004. Reward expectation, orientation of attention and locus coeruleus-medial frontal cortex interplay during learning. Eur $J$ Neurosci 20: 791-802.

Bradfield LA, Bertran-Gonzalez J, Chieng B, Balleine BW. 2013. The thalamostriatal pathway and cholinergic control of goal-directed action: interlacing new with existing learning in the striatum. Neuron 79: $153-166$.

Braun S, Hauber W. 2011. The dorsomedial striatum mediates flexible choice behavior in spatial tasks. Behav Brain Res 220: 288-293.

Brown HD, Baker PM, Ragozzino ME. 2010. The parafascicular thalamic nucleus concomitantly influences behavioral flexibility and dorsomedial striatal acetylcholine output in rats. J Neurosci 30: 14390-14398

Canteras NS, Shammah-Lagnado SJ, Silva BA, Ricardo JA. 1990. Afferent connections of the subthalamic nucleus: a combined retrograde and anterograde horseradish peroxidase study in the rat. Brain Res 513: $43-59$.

Castane A, Theobald DE, Robbins TW. 2010. Selective lesions of the dorsomedial striatum impair serial spatial reversal learning in rats. Behav Brain Res 210: 74-83.

Castle M, Aymerich MS, Sanchez-Escobar C, Gonzalo N, Obeso JA Lanciego JL. 2005. Thalamic innervation of the direct and indirect basal ganglia pathways in the rat: ipsi- and contralateral projections. J Comp Neurol 483: $143-153$.

Chudasama Y, Baunez C, Robbins TW. 2003. Functional disconnection of the medial prefrontal cortex and subthalamic nucleus in attentional performance: evidence for corticosubthalamic interaction. J Neurosci 23: $5477-5485$

Conde F, Maire-Lepoivre E, Audinat E, Crepel F. 1995. Afferent connections of the medial frontal cortex of the rat. II. Cortical and subcortical afferents. J Comp Neurol 352: 567-593.

Dias R, Aggleton JP. 2000. Effects of selective excitotoxic prefrontal lesions on acquisition of nonmatching- and matching-to-place in the T-maze in the rat: differential involvement of the prelimbic-infralimbic and anterior cingulate cortices in providing behavioural flexibility. Eur J Neurosci 12: 4457-4466.

Ding JB, Guzman JN, Peterson JD, Goldberg JA, Surmeier DJ. 2010. Thalamic gating of corticostriatal signaling by cholinergic interneurons. Neuron 67: 294-307.

Dunnett SB, Meldrum A, Muir JL. 2005. Frontal-striatal disconnection disrupts cognitive performance of the frontal-type in the rat. Neuroscience 135: 1055-1065.

Enomoto T, Tse MT, Floresco SB. 2011. Reducing prefrontal $\gamma$-aminobutyric acid activity induces cognitive, behavioral, and dopaminergic abnormalities that resemble schizophrenia. Biol Psychiatry 69: $432-441$.

Featherstone RE, McDonald RJ. 2005. Lesions of the dorsolateral striatum impair the acquisition of a simplified stimulus-response dependent conditional discrimination task. Neuroscience 136: 387-395.

Floresco SB, Ghods-Sharifi S, Vexelman C, Magyar O. 2006a. Dissociable roles for the nucleus accumbens core and shell in regulating set shifting. J Neurosci 26: 2449-2457.

Floresco SB, Magyar O, Ghods-Sharifi S, Vexelman C, Tse MT. 2006b. Multiple dopamine receptor subtypes in the medial prefrontal cortex of the rat regulate set-shifting. Neuropsychopharmacology 31: 297-309.

Floresco SB, Block AE, Tse MT. 2008. Inactivation of the medial prefrontal cortex of the rat impairs strategy set-shifting, but not reversal learning, using a novel, automated procedure. Behav Brain Res 190: 85-96.

Fujisawa S, Buzsaki G. 2011. A 4 Hz oscillation adaptively synchronizes prefrontal, VTA, and hippocampal activities. Neuron 72: 153-165.

Gabbott PL, Warner TA, Jays PR, Salway P, Busby SJ. 2005. Prefrontal cortex in the rat: projections to subcortical autonomic, motor, and limbic centers. J Comp Neurol 492: 145-177.

Gruber AJ, O'Donnell P. 2009. Bursting activation of prefrontal cortex drives sustained up states in nucleus accumbens spiny neurons in vivo. Synapse 63: 173-180.

Gruber AJ, Solla SA, Surmeier DJ, Houk JC. 2003. Modulation of striatal single units by expected reward: a spiny neuron model displaying dopamine-induced bistability. I Neurophysiol 90: 1095-1114.

Hallock HL, Arreola AC, Shaw CL, Griffin AL. 2013. Dissociable roles of the dorsal striatum and dorsal hippocampus in conditional discrimination and spatial alternation T-maze tasks. Neurobiol Learn Mem 100: $108-116$.

Hikosaka O, Isoda M. 2010. Switching from automatic to controlled behavior: cortico-basal ganglia mechanisms. Trends Cogn Sci 14: $154-161$.

Hikosaka O, Wurtz RH. 1985. Modification of saccadic eye movements by GABA-related substances. I. Effect of muscimol and bicuculline in monkey superior colliculus. J Neurophysiol 53: 266-291.

Höhn S, Dallérac G, Faure A, Urbach YK, Nguyen HP, Riess O, von Hörsten S, Le Blanc P, Desvignes N, El Massioui N. 2011. Behavioral and in vivo electrophysiological evidence for presymptomatic alteration of prefrontostriatal processing in the transgenic rat model for Huntington disease. J Neurosci 31: 8986-8997.

Horst NK, Laubach M. 2012. Working with memory: evidence for a role for the medial prefrontal cortex in performance monitoring during spatial delayed alternation. J Neurophysiol 108: 3276-3288.

Hyafil A, Summerfield C, Koechlin E. 2009. Two mechanisms for task switching in the prefrontal cortex. J Neurosci 29: 5135-5142.

Kawabe T, Chitravanshi VC, Kawabe K, Sapru HN. 2008. Cardiovascular function of a glutamatergic projection from the hypothalamic paraventricular nucleus to the nucleus tractus solitarius in the rat. Neuroscience 153: 605-617.

Kim J, Ragozzino ME. 2005. The involvement of the orbitofrontal cortex in learning under changing task contingencies. Neurobiol Learn Mem 83: $125-133$.

Kimchi EY, Laubach M. 2009. The dorsomedial striatum reflects response bias during learning. J Neurosci 29: 14891-14902.

Konishi S, Chikazoe J, Jimura K, Asari T, Miyashita Y. 2005. Neural mechanism in anterior prefrontal cortex for inhibition of prolonged set interference. Proc Natl Acad Sci 102: 12584-12588.

Krupa DJ, Ghazanfar AA, Nicolelis MA. 1999. Immediate thalamic sensory plasticity depends on corticothalamic feedback. Proc Natl Acad Sci 96 8200-8205.

Lee I, Lee SH. 2013. Putting an object in context and acting on it: neural mechanisms of goal-directed response to contextual object. Rev Neurosci 24: 27-49.

Leenaars CH, Joosten RN, Zwart A, Sandberg H, Ruimschotel E, Hanegraaf MA, Dematteis M, Feenstra MG, van Someren EJ. 2012. Switch-task performance in rats is disturbed by $12 \mathrm{~h}$ of sleep deprivation but not by $12 \mathrm{~h}$ of sleep fragmentation. Sleep 35: 211-221.

Mailly P, Aliane V, Groenewegen HJ, Haber SN, Deniau JM. 2013. The rat prefrontostriatal system analyzed in 3D: evidence for multiple interacting functional units. J Neurosci 33: 5718-5727.

McCool MF, Patel S, Talati R, Ragozzino ME. 2008. Differential involvement of M1-type and M4-type muscarinic cholinergic receptors in the dorsomedial striatum in task switching. Neurobiol Learn Mem 89: $114-124$.

McDonald RJ, King AL, Foong N, Rizos Z, Hong NS. 2008. Neurotoxic lesions of the medial prefrontal cortex or medial striatum impair multiple-location place learning in the water task: evidence for neural structures with complementary roles in behavioural flexibility. Exp Brain Res 187: 419-427.

McMullan S, Pilowsky PM. 2012. Sympathetic premotor neurones project to and are influenced by neurones in the contralateral rostral ventrolateral medulla of the rat in vivo. Brain Res 1439: 34-43. 
Mohler EG, Baker PM, Gannon KS, Jones SS, Shacham S, Sweeney JA, Ragozzino ME. 2012. The effects of PRX-07034, a novel 5-HT 6 antagonist, on cognitive flexibility and working memory in rats. Psychopharmacology (Berl) 220: 687-696.

Narayanan NS, Laubach M. 2006. Top-down control of motor cortex ensembles by dorsomedial prefrontal cortex. Neuron 52: 921-931.

Narayanan NS, Laubach M. 2009. Delay activity in rodent frontal cortex during a simple reaction time task. J Neurophysiol 101: $2859-2871$.

Nicolle MM, Baxter MG. 2003. Glutamate receptor binding in the frontal cortex and dorsal striatum of aged rats with impaired attentional set-shifting. Eur J Neurosci 18: 3335-3342.

Oualian C, Gisquet-Verrier P. 2010. The differential involvement of the prelimbic and infralimbic cortices in response conflict affects behavioral flexibility in rats trained in a new automated strategy-switching task. Learn Mem 17: 654-668.

Palencia CA, Ragozzino ME. 2004. The influence of NMDA receptors in the dorsomedial striatum on response reversal learning. Neurobiol Learn Mem 82: 81-89.

Palencia CA, Ragozzino ME. 2006. The effect of $N$-methyl-D-aspartate receptor blockade on acetylcholine efflux in the dorsomedial striatum during response reversal learning. Neuroscience 143: 671-678.

Pastuzyn ED, Chapman DE, Wilcox KS, Keefe KA. 2012. Altered learning and Arc-regulated consolidation of learning in striatum by methamphetamine-induced neurotoxicity. Neuropsychopharmacology 37: $885-895$.

Paxinos G, Watson C. 1997. The rat brain in stereotaxic coordinates. Academic Press, San Diego, CA.

Pisa M, Cyr J. 1990. Regionally selective roles of the rat's striatum in modality-specific discrimination learning and forelimb reaching. Behav Brain Res 37: 281-292.

Ragozzino ME. 2003. Acetylcholine actions in the dorsomedial striatum support the flexible shifting of response patterns. Neurobiol Learn Mem 80: $257-267$.

Ragozzino ME, Rozman S. 2007. The effect of rat anterior cingulate inactivation on cognitive flexibility. Behav Neurosci 121: 698-706.
Ragozzino ME, Detrick S, Kesner RP. 1999a. Involvement of the prelimbicinfralimbic areas of the rodent prefrontal cortex in behavioral flexibility for place and response learning. J Neurosci 19: 4585-4594.

Ragozzino ME, Wilcox C, Raso M, Kesner RP. 1999b. Involvement of rodent prefrontal cortex subregions in strategy switching. Behav Neurosci 113: $32-41$.

Ragozzino ME, Ragozzino KE, Mizumori SJ, Kesner RP. 2002. Role of the dorsomedial striatum in behavioral flexibility for response and visual cue discrimination learning. Behav Neurosci 116: 105-115.

Ragozzino ME, Kim J, Hassert D, Minniti N, Kiang C. 2003. The contribution of the rat prelimbic-infralimbic areas to different forms of task switching. Behav Neurosci 117: 1054-1065.

Rich EL, Shapiro ML. 2007. Prelimbic/infralimbic inactivation impairs memory for multiple task switches, but not flexible selection of familiar tasks. J Neurosci 27: 4747-4755.

Rich EL, Shapiro M. 2009. Rat prefrontal cortical neurons selectively code strategy switches. J Neurosci 29: 7208-7219.

Seo M, Lee E, Averbeck BB. 2012. Action selection and action value in frontal-striatal circuits. Neuron 74: 947-960.

Sesack SR, Deutch AY, Roth RH, Bunney BS. 1989. Topographical organization of the efferent projections of the medial prefrontal cortex in the rat: $\mathrm{n}$ anterograde tract-tracing study with Phaseolus vulgaris leucoagglutinin. J Comp Neurol 290: 213-242.

Stefani MR, Groth K, Moghaddam B. 2003. Glutamate receptors in the rat medial prefrontal cortex regulate set-shifting ability. Behav Neurosci 117: $728-737$.

Tzavos A, Jih J, Ragozzino ME. 2004. Differential effects of M1 muscarinic receptor blockade and nicotinic receptor blockade in the dorsomedial striatum on response reversal learning. Behav Brain Res 154: 245-253.

van Schouwenburg M, Aarts E, Cools R. 2010. Dopaminergic modulation of cognitive control: distinct roles for the prefrontal cortex and the basal ganglia. Curr Pharm Des 16: 2026-2032.

Wolfensteller U, Ruge H. 2012. Frontostriatal mechanisms in instruction-based learning as a hallmark of flexible goal-directed behavior. Front Psychol 3: 192.

Received February 11, 2014; accepted in revised form June 10, 2014. 


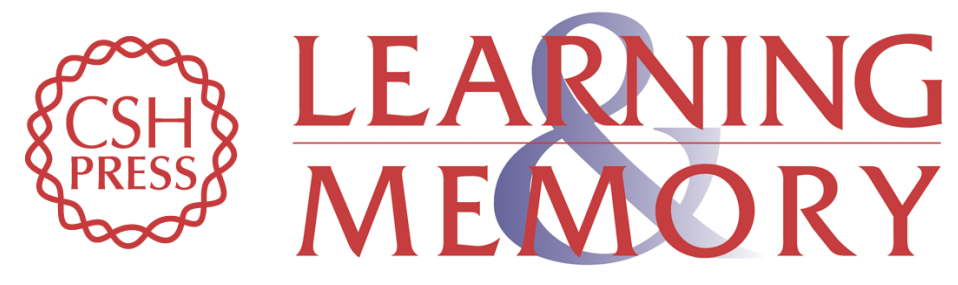

\section{Contralateral disconnection of the rat prelimbic cortex and dorsomedial striatum impairs cue-guided behavioral switching}

Phillip M. Baker and Michael E. Ragozzino

Learn. Mem. 2014, 21:

Access the most recent version at doi:10.1101/lm.034819.114

\section{Supplemental http://learnmem.cshlp.org/content/suppl/2014/07/09/21.8.368.DC1 Material}

References This article cites 68 articles, 14 of which can be accessed free at: http://learnmem.cshlp.org/content/21/8/368.full.html\#ref-list-1

Creative This article is distributed exclusively by Cold Spring Harbor Laboratory Press for the Commons first 12 months after the full-issue publication date (see

License http://learnmem.cshlp.org/site/misc/terms.xhtml). After 12 months, it is available under a Creative Commons License (Attribution-NonCommercial 4.0 International), as described at http://creativecommons.org/licenses/by-nc/4.0/.

Email Alerting Receive free email alerts when new articles cite this article - sign up in the box at the Service top right corner of the article or click here. 